\title{
Floristic composition, biological spectrum and phenological pattern of vegetation in the subtropical forest of Kotli District, AJK, Pakistan
}

\author{
Muhammad Shoaib Amjad ${ }^{1,2^{*}}$, Muhammad Arshad ${ }^{1}$, Susan Page ${ }^{3}$, \\ Rahmatullah Qureshi ${ }^{1}$ and Sarwat Naz Mirza ${ }^{4}$ \\ 1. Department of Botany, PMAS-University of Arid Agriculture Rawalpindi-Pakistan \\ 2. Department of Botany, Women University of Azad Jammu \& Kashmir Bagh-Pakistan \\ 3. Department of Geography, University of Leicester, UK \\ 4. Department of Forestry, PMAS-University of Arid Agriculture, Rawalpindi-Pakistan \\ *Corresponding author's email: malikshoaib1165@yahoo.com \\ Citation
}

Muhammad Shoaib Amjad, Muhammad Arshad, Susan Page, Rahmatullah Qureshi and Sarwat Naz Mirza. Floristic composition, biological spectrum and phenological pattern of vegetation in the subtropical forest of Kotli District, AJK, Pakistan. Pure and Applied Biology. Vol. 6, Issue 2, pp426-447. http://dx.doi.org/10.19045/bspab.2017.60043

Received: 16/08/2016

Revised: 22/02/2017

Accepted: 20/03/2017

Online First: 25/03/2017

\section{Abstract}

A comprehensive floristic survey of Kotli District, Azad Jammu and Kashmir was conducted during 2014-2016 to analyse phytodiversity and ecological characteristics of plant resources. The flora comprised of 202 plant species distributed among 71 families and 176 genera. Of these, 6 species were pteridophytes, 1 species was a gymnosperm, 159 species were dicotyledons and 36 species were monocotyledons. Based on species numbers, Asteraceae (23 Spp), Poaceae (20 Spp), Fabaceae (15 Spp), Labiatae (11 Spp), and Euphorbiaceae (07 Spp) were the leading families. Based on biological spectrum, therophytes (73 Spp., $36.14 \%$ ) were dominant followed by hemicryptophytes (19.31\%), nanophanerophytes (13.37\%), geophytes (10.40\%), lianas (7.92\%), chamaephytes (7.43\%) and megaphanerophytes (5.45\%). Nanophylls (36.14\%), leptophylls $(26.73 \%)$ and microphylls $(26.24 \%)$ were the major leaf size classes. Phenological study revealed four different flowering seasons, with April the peak flowering month during which 122 species blossomed. This current study will provide essential baseline information for ecologists, taxonomists and conservationists to carry out more detailed ecological investigations and conservation management planning for the plant resources of this area.

Keywords: Floristic list; Life form; Leaf spectra; Phenology; Kotli

\section{Introduction}

The diversity and ecological characteristics of the plants of a particular area depend upon environmental conditions, including altitude and climate. Thus ecological characteristics, such as life form, leaf spectra and phenological pattern, can be used as indicators of prevailing environmental conditions [1]. Flora refers to all plant life occurring in any particular geographic region at a specific geological period and includes the number of species. In contrast 
vegetation refers to the number and distribution of individuals of different plant species in a particular ecosystem [2]. The study of floristic composition is a common taxonomic practice as it provides the baseline information for subsequent, more detailed ecological investigation as well as planning for conservation and sustainable management of the resources of the area [1, 3]. The concept of life form was first introduced by Humboldt with the term Vegetative Form. It ranked next to floristic composition in ecological studies [4] and is the outcome of the adaptation of plants to certain climatic conditions [5]. The life form of a plant reflects the climate of the area and is also useful in comparing the geographical distribution of plant communities. Traditionally it was used in the description of vegetation structure at the community level [6]. The most compact and consistent classification of life form is that of Raunkiaer, which is based on the degree of presence and protection of perenating buds. On the basis of life form, he recognized three different phytoclimates on earth including the therophytic climate in deserts, hemicryptophytic in the temperate zone and phanerophytic in the tropics. MuellerDumbois and Ellenberg [7] divided plant species in to five main life form categories based on similarity in their structure and function. Similarly leaf size classes have been used in vegetation description and in order to understand the physiological processes of plants and plant communities [8]. Phenology refers to the study of the timing and causes of repeated biological phenomena with respect to the non-living and living factors and the relationship between the phases of different or the same species [9, 10]. Small variations in climate can have a great effect on the vegetation [11]. For example, vegetation can be characterized by different patterns of climate-induced phenological events [12], with flowering induced by significant rain in the months of winter and summer, temperature differences, changes in photoperiodism, and drought [13]. In addition to characterizing the vegetation, such studies can also be useful in developing effective management strategies as well as better understanding community level interactions and the vegetation regeneration potential [14].

Some previous evaluation studies have been carried out on the floristic composition and ecological characteristics and phenological patterns of the vegetation of the more remote regions of Pakistan, including Azad Kashmir [1, 3, 15-23]. However, the inaccessibility of the Kotli District means that this area has received very little exploration by plant taxonomists and ecologists, with only a very few published studies [23-28]. Therefore, the present work was conducted in order to report on this areas floristic diversity and its ecological characteristics in order to provide the baseline information for subsequent more detailed ecological investigations and to provide the basis for conservation management of the natural resources of the area.

\section{Materials and methods}

\section{Study area, vegetation and climate}

Kotli is one of the biodiversity rich areas of Azad Jammu and Kashmir. The district is located $130 \mathrm{~km}$ north of Islamabad, the capital of Pakistan $\left(73^{\circ} .47 .180^{\prime} \mathrm{E}\right.$ to $74^{\circ}$ $04.613^{\prime} \mathrm{E}$ longitude; $33^{\circ} 23.069^{\prime} \mathrm{N}$ to $33^{\circ}$ $29.344^{\prime} \mathrm{N}$ latitude; altitudinal range $450 \mathrm{~m}$ to $1900 \mathrm{~m}$ ). Figure 1 shows the location of the various sampling sites used in this study. The region is mountainous and covers an area of $1860 \mathrm{~km}^{2}$. Climatically the area is dry subtropical at the lower altitudes and subtropical humid in the upper reaches with a mean annual precipitation of $1250 \mathrm{~mm}$. Three different forest types have been recognized in the area, namely subtropical 
scrub forest, subtropical chir pine forest and subtropical broad leaf humid forest. The highest average monthly precipitation is recorded during the month of July (291 $\mathrm{mm}$ ), while the lowest average monthly precipitation of $19 \mathrm{~mm}$ occurs during the month of November. Temperatures reach a peak during June and July, with average daily minimum temperatures of $23.5^{\circ} \mathrm{C}$ and $23.7^{\circ} \mathrm{C}$ respectively and maximum temperatures of $37.1^{\circ} \mathrm{C}$ and $33.9^{\circ} \mathrm{C}$

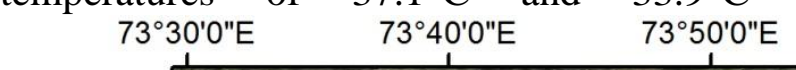

Figure 1. Shows the location of the various sampling sites used in this study

\section{Field surveys and plant collection}

Field surveys were conducted throughout the district during 2014-2016. Plants were collected in triplicate, dried and mounted on herbarium sheets. The plants were identified by using flora of Pakistan [29, 30]. The correct names were obtained from tropicos (www.tropicos.org) and the international

respectively, while the coldest months are December and January with mean minimum temperatures of $4.8{ }^{\circ} \mathrm{C}$ and $3.9{ }^{\circ} \mathrm{C}$ respectively and mean maximum temperatures of $20.8^{\circ} \mathrm{C}$ and $18.6^{\circ} \mathrm{C}$ respectively. The mean maximum relative humidity of $68.2 \%$ occurs during August and the minimum relative humidity of $33.2 \%$ occurs during March (Source: Pakistan Meteorological Department,

Lahore).

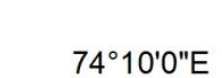


Agriculture University Rawalpindi, Pakistan.

\section{Floristic list, life form and leaf spectra}

A complete list of plant species was prepared by alphabetical arrangement of the plant families and then in each plant family different plant species were arranged alphabetically. Plants were classified in to different life form and leaf size classes following Raunkiaer (1934), Hussain (1989), Badsha et al (2013) and Ali et al. (2016) $[3,6,16,31]$. For quick estimation of leaf size in the field, the diagram of Raukiaer (1934) was used.

Phenology
The phenological observations of plants were recorded monthly from August 2014 to July 2016. Starting month and duration of strobili development, sporogenesis and flowering was recorded for each plant species. A Microsoft excel sheet was prepared by giving value 1 when the species was found in a flowering or reproductive stage in a particular month and 0 other wise. The sheet was than exported into PC ORD version 5 [32, 33]. Cluster analysis was performed to merge the months in to four different flowering seasons. The percentage of flowering within each month was calculated by applying the formula

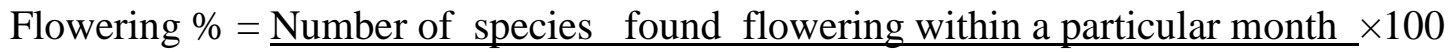

\section{Total number of Species}

\section{Results}

The detailed floristic checklist is given in Appendix I which includes family, botanical name, life form, leaf spectra, seasonality and phenology of plant species.

\section{Appendix I. Floristic composition, Biological Spectrum and phenology of plant species} recorded from District Kotli, Azad Jammu \& Kashmir

\begin{tabular}{|c|c|c|c|c|c|c|c|}
\hline \multirow{2}{*}{ Division/Family } & \multirow{2}{*}{ S. No } & \multirow{2}{*}{ Species } & \multicolumn{2}{|c|}{ Seasonality } & \multirow{2}{*}{$\begin{array}{l}\text { Life } \\
\text { Form }\end{array}$} & \multirow{2}{*}{$\begin{array}{l}\text { Leaf } \\
\text { Spectra }\end{array}$} & \multirow[t]{2}{*}{ Phenology } \\
\hline & & & Monsoon & Spring & & & \\
\hline \multicolumn{8}{|c|}{ A. Pteridophytes } \\
\hline Dryopteridaceae & 1. & Dryopteris stewartii Fraser-Jenk. & + & + & $\mathrm{G}$ & $\mathrm{Me}$ & May-Sep \\
\hline \multirow{4}{*}{ Pteridaceae } & 2. & Adiantum incisum Forssk. & + & + & G & $\mathrm{N}$ & Jun-Oct \\
\hline & 3. & Adiantum venustum $\mathrm{D}$. Don & + & - & G & $\mathrm{N}$ & Jun-Aug \\
\hline & 4. & $\begin{array}{l}\text { Onychium japonicum (Thunb.) } \\
\text { Kunze }\end{array}$ & + & - & G & $\mathrm{L}$ & Jun-Sep \\
\hline & 5. & Pteris cretica $\mathrm{L}$. & + & + & G & $\mathrm{Mi}$ & Jun-Aug \\
\hline Selaginellaceae & 6. & $\begin{array}{l}\text { Selaginella chrysocaulos (Hook. \& } \\
\text { Grev.) Spring }\end{array}$ & + & - & G & $\mathrm{L}$ & May-Aug \\
\hline \multicolumn{8}{|c|}{ B. Gymnosperms } \\
\hline Pinaceae & 7. & Pinus roxburghii Sargent & + & + & MP & $\mathrm{L}$ & Mar-Apr \\
\hline \multicolumn{8}{|c|}{ C. Angiosperms } \\
\hline \multicolumn{8}{|c|}{ a. Monocotyledons } \\
\hline Araceae & 8. & $\begin{array}{l}\text { Sauromatum venosum (Aiton) } \\
\text { Kunth }\end{array}$ & + & - & G & $\mathrm{Me}$ & Apr-May \\
\hline \multirow[t]{2}{*}{ Asparagaceae } & 9. & $\begin{array}{l}\text { Asparagus capitatus subsp. gracilis } \\
\text { (Royle ex Baker) Browicz }\end{array}$ & + & - & NP & $\mathrm{L}$ & Mar-Sep \\
\hline & 10. & Drimia indica (Roxb.) Jessop & + & - & $\mathrm{G}$ & $\overline{\mathrm{L}}$ & Apr-May \\
\hline Colchicaceae & 11. & Gloriosa superba L. & - & + & $\mathrm{L}$ & Mi & Jul-Sep \\
\hline Commelinaceae & 12. & Commelina benghalensis $\mathrm{L}$. & + & + & Th & Mi & Aug-Nov \\
\hline \multirow{4}{*}{ Cyperaceae } & 13. & Carex sempervirens Vill. & + & + & $\mathrm{H}$ & $\mathrm{L}$ & Apr-Jul \\
\hline & 14. & Cyperus difformis $\mathrm{L}$. & + & - & $\mathrm{G}$ & $\mathrm{N}$ & Jul-Sep \\
\hline & 15. & Cyperus niveus Retz. & + & + & $\mathrm{G}$ & $\mathrm{L}$ & Apr-Aug \\
\hline & 16. & Cyperus rotundus $\mathrm{L}$. & + & - & $\mathrm{G}$ & $\mathrm{N}$ & Apr-Oct \\
\hline
\end{tabular}




\begin{tabular}{|c|c|c|c|c|c|c|c|}
\hline & 17. & Erioscirpus comosus (Nees) Palla & + & + & $\mathrm{G}$ & $\mathrm{L}$ & Mar-Apr \\
\hline \multirow[b]{2}{*}{ Dioscoreaceae } & 18. & Dioscorea bulbifera L. & + & - & $\mathrm{L}$ & $\mathrm{Me}$ & Jul-Sep \\
\hline & 19. & $\begin{array}{l}\text { Dioscorea melanophyma Prain \& } \\
\text { Burkill }\end{array}$ & + & - & $\mathrm{L}$ & $\mathrm{Me}$ & Aug-Oct \\
\hline Juncaceae & 20. & Juncus articulatus L. & + & - & $\mathrm{G}$ & $\mathrm{L}$ & Apr-May \\
\hline Liliaceae & 21. & Gagea pakistanica Levichev \& Ali & - & + & $\mathrm{G}$ & $\mathrm{L}$ & Mar-Apr \\
\hline Orchidaceae & 22. & Zeuxine strateumatica (L.) Schltr. & + & - & Th & $\mathrm{Mi}$ & Mar-Apr \\
\hline \multirow{20}{*}{ Poaceae } & 23. & Aristida adscensionis L. & + & - & Th & $\mathrm{Mi}$ & Jul-Oct \\
\hline & 24. & $\begin{array}{l}\text { Brachiaria eruciformis (Sm.) } \\
\text { Griseb. }\end{array}$ & - & + & Th & $\mathrm{L}$ & Jul-Sep \\
\hline & 25. & $\begin{array}{l}\text { Brachiaria reptans (L.) C.A. } \\
\text { Gardner \& C.E. Hubb. }\end{array}$ & + & + & Th & $\mathrm{L}$ & Jun-Nov \\
\hline & 26. & Cenchrus ciliaris L. & - & + & $\mathrm{H}$ & $\mathrm{L}$ & Feb-Mar \\
\hline & 27. & Chrysopogon aucheri (Boiss.) Stapf & + & + & $\mathrm{H}$ & $\mathrm{L}$ & Mar-May \\
\hline & 28. & Cynodon dactylon (L.) Pers. & + & + & $\mathrm{H}$ & $\mathrm{L}$ & $\begin{array}{l}\text { Round the } \\
\text { year }\end{array}$ \\
\hline & 29. & $\begin{array}{l}\text { Dactyloctenium aegyptium (L.) } \\
\text { Willd. }\end{array}$ & & + & Th & $\mathrm{Mi}$ & Jul-Oct \\
\hline & 30. & $\begin{array}{l}\text { Dichanthium annulatum (Forssk.) } \\
\text { Stapf }\end{array}$ & + & + & $\mathrm{H}$ & $\mathrm{N}$ & Mar-Oct \\
\hline & 31. & Digitaria setigera Roth & - & + & Th & $\mathrm{L}$ & Sep-Oct \\
\hline & 32. & Eleusine indica (L.) Gaertn. & - & + & Th & $\mathrm{L}$ & Jun-Aug \\
\hline & 33. & Eragrostis japonica (Thunb.) Trin. & + & - & Th & $\mathrm{N}$ & Aug-Oct \\
\hline & 34. & $\begin{array}{l}\text { Heteropogon contortus (L.) P. } \\
\text { Beauv. ex Roem. \& Schult. }\end{array}$ & + & + & $\mathrm{H}$ & $\mathrm{N}$ & Sep-Nov \\
\hline & 35. & Imperata cylindrica (L.) P. Beauv. & + & + & $\mathrm{G}$ & $\mathrm{L}$ & Apr-Aug \\
\hline & 36. & $\begin{array}{l}\text { Oplismenus undulatifolius (Ard.) P. } \\
\text { Beauv. }\end{array}$ & - & + & $\mathrm{H}$ & $\mathrm{L}$ & Aug-Sep \\
\hline & 37. & Poa annua L. & - & + & Th & $\mathrm{L}$ & Apr-Sep \\
\hline & 38. & Saccharum spontaneum L. & + & + & $\mathrm{Ch}$ & $\mathrm{L}$ & Jul-Sep \\
\hline & 39. & $\begin{array}{l}\text { Setaria pumila (Poir.) Roem. \& } \\
\text { Schult. }\end{array}$ & + & + & Th & $\mathrm{N}$ & Jun-Oct \\
\hline & 40. & Setaria viridis (L.) P. Beauv. & + & + & Th & $\mathrm{N}$ & Jun-Sep \\
\hline & 41. & Sorghum halepense (L.) Pers. & + & + & G & $\mathrm{N}$ & May-Oct \\
\hline & 42. & $\begin{array}{l}\text { Themeda anathera (Nees ex Steud.) } \\
\text { Hack. }\end{array}$ & + & + & $\mathrm{H}$ & $\mathrm{L}$ & Jun-Oct \\
\hline Smilacaceae & 43. & Smilax glaucophylla Klotzsch & + & + & $\mathrm{L}$ & $\mathrm{Mi}$ & Apr-May \\
\hline \multicolumn{8}{|c|}{ b. Dicotyledons } \\
\hline \multirow{5}{*}{ Acanthaceae } & 44. & Barleria cristata $\mathrm{L}$. & + & - & $\mathrm{Ch}$ & $\mathrm{Mi}$ & Nov-Feb \\
\hline & 45. & Dicliptera bupleuroides Nees & + & + & Th & $\mathrm{L}$ & Jun-Oct \\
\hline & 46. & Justicia adhatoda L. & + & + & NP & $\mathrm{Me}$ & Jul-Oct \\
\hline & 47. & $\begin{array}{l}\text { Justicia peploides (Nees) T. } \\
\text { Anderson }\end{array}$ & + & - & Th & $\mathrm{N}$ & Jul-Sep \\
\hline & 48. & $\begin{array}{l}\text { Pteracanthus alatus (Wall. ex Nees) } \\
\text { Bremek. }\end{array}$ & + & - & Th & Mi & Jul-Sep \\
\hline Adoxaceae & 49. & $\begin{array}{l}\text { Viburnum grandiflorum Wallich ex } \\
\text { DC }\end{array}$ & + & + & NP & $\mathrm{Me}$ & Nov-Jun \\
\hline Aizoaceae & 50. & Zaleya pentandra (L.) C. Jeffrey & + & - & $\mathrm{Ch}$ & $\mathrm{N}$ & Apr-Jul \\
\hline \multirow{5}{*}{ Amaranthaceae } & 51. & Achyranthes aspera L. & + & + & Th & $\mathrm{N}$ & Mar-Aug \\
\hline & 52. & Alternanthera pungens Kunth & + & - & Th & $\mathrm{N}$ & Jun-Oct \\
\hline & 53. & Amaranthus viridis $\mathrm{L}$. & + & + & Th & $\mathrm{N}$ & $\begin{array}{l}\text { Round the } \\
\text { year }\end{array}$ \\
\hline & 54. & Chenopodium album L. & + & - & Th & $\mathrm{N}$ & Jan-Sep \\
\hline & 55. & Pupalia lappacea (L.) Juss. & - & + & $\mathrm{Ch}$ & $\mathrm{N}$ & Apr-Nov \\
\hline
\end{tabular}


Amjad et al.

\begin{tabular}{|c|c|c|c|c|c|c|c|}
\hline Anacardiaceae & 56. & Cotinus coggygria Scop. & + & + & NP & $\mathrm{Mi}$ & Apr-May \\
\hline \multirow{5}{*}{ Apiaceae } & 57. & Aegopodium podagrari $\mathrm{L}$. & + & - & Th & $\mathrm{Mi}$ & Feb-May \\
\hline & 58. & Bupleurum falcatum $\mathrm{L}$. & + & - & Th & $\mathrm{L}$ & Jun-Oct \\
\hline & 59. & Heracleum candicans Wall. ex DC. & + & & $\mathrm{Ch}$ & $\mathrm{Me}$ & Jun-Sep \\
\hline & 60. & Scandix pecten-veneris L. & - & + & Th & $\mathrm{L}$ & Mar-May \\
\hline & 61. & Torilis nodosa (L.) Gaertn. & - & + & Th & $\mathrm{L}$ & Mar-May \\
\hline \multirow{3}{*}{ Apocynaceae } & 62. & Carissa opaca Stapf ex Haines & + & + & NP & Mi & Apr-Jun \\
\hline & 63. & Nerium oleander L. & + & + & NP & $\mathrm{Mi}$ & Apr-Sep \\
\hline & 64. & Tylophora hirsuta (Wall.) Wight & + & - & $\mathrm{L}$ & $\mathrm{Me}$ & Jul-Sep \\
\hline Araliaceae & 65. & Hedera nepalensis K. Koch. & + & + & $\mathrm{L}$ & Mi & Jul-Sep \\
\hline \multirow{23}{*}{ Asteraceae } & 66. & Achillea millefolium $\mathrm{L}$. & + & + & $\mathrm{H}$ & $\mathrm{L}$ & Jun-Sep \\
\hline & 67. & Adenostemma lavenia (L.) Kuntze & + & - & Th & Mi & Apr-May \\
\hline & 68. & $\begin{array}{l}\text { Anaphalis margaritacea (L.) Benth. } \\
\text { and Hook.f. }\end{array}$ & + & - & G & $\mathrm{N}$ & Jul-Sep \\
\hline & 69. & Artemisia scoparia Waldst. \& Kit. & + & + & $\mathrm{Ch}$ & $\mathrm{L}$ & Jul-Nov \\
\hline & 70. & Bidens bipinnata $\mathrm{L}$. & - & + & $\mathrm{H}$ & Mi & Dec-Mar \\
\hline & 71. & $\begin{array}{l}\text { Bidens biternata (Lour.) Merr. \& } \\
\text { Sherff }\end{array}$ & + & + & Th & $\mathrm{N}$ & Dec-Mar \\
\hline & 72. & Carpesium cernuит $\mathrm{L}$. & + & + & $\mathrm{Th}$ & $\mathrm{Mi}$ & Jul-Sep \\
\hline & 73. & Cirsium wallichii DC. & + & + & $\mathrm{H}$ & $\mathrm{N}$ & Apr-Jun \\
\hline & 74. & Conyza bonariensis (L.) Cronq. & + & + & Th & $\mathrm{N}$ & Mar-Jun \\
\hline & 75. & Gerbera gossypina (Royle) Beauv. & + & + & Th & $\mathrm{Mi}$ & May-Jul \\
\hline & 76. & Lactuca dissecta D. Don & - & + & $\mathrm{H}$ & $\mathrm{N}$ & Mar-May \\
\hline & 77. & $\begin{array}{l}\text { Launaea procumbens (Roxb.) } \\
\text { Ramayya \& Rajagopal }\end{array}$ & + & - & $\mathrm{Ch}$ & $\mathrm{Me}$ & Mar-Apr \\
\hline & 78. & Myriactis nepalensis Less.. & + & - & Th & $\mathrm{N}$ & Sep-Oct \\
\hline & 79. & Parthenium hysterophorus L. & + & + & Th & $\mathrm{N}$ & Apr-Oct \\
\hline & 80. & $\begin{array}{l}\text { Saussurea heteromalla (D. Don) } \\
\text { Handel-Mazzetti }\end{array}$ & + & - & $\mathrm{H}$ & $\mathrm{Mi}$ & Mar-Aug \\
\hline & 81. & $\begin{array}{l}\text { Senecio nudicaulis Buchanan- } \\
\text { Hamilton ex D. Don }\end{array}$ & - & + & G & Mi & Apr-May \\
\hline & 82. & Serratula praealta $\mathrm{L}$. & - & + & $\mathrm{H}$ & $\mathrm{N}$ & Jul-Sep \\
\hline & 83. & Sonchus arvensis L. & - & + & Th & $\mathrm{N}$ & Feb-May \\
\hline & 84. & Sonchus asper (L.) Hill & + & + & Th & $\mathrm{Me}$ & Mar-Jun \\
\hline & 85. & Taraxacum officinale F.H. Wigg. & + & + & $\mathrm{G}$ & $\mathrm{Mi}$ & Feb-Apr \\
\hline & 86. & Tridax procumbens $\mathrm{L}$. & + & + & $\mathrm{H}$ & $\mathrm{Mi}$ & Sep-Jan \\
\hline & 87. & Vernonia cinerea $(\mathrm{L}$.$) Less.$ & + & + & Th & $\mathrm{L}$ & Mar-Jun \\
\hline & 88. & Youngia japonica (L.) DC. & - & + & Th & $\mathrm{N}$ & Mar-May \\
\hline Balsaminaceae & 89. & Impatiens edgeworthii Hook. f. & - & + & $\mathrm{Th}$ & Mi & Jul-Sep \\
\hline Berberidaceae & 90. & Berberis lycium Royle & + & + & NP & $\mathrm{N}$ & Apr-Jun \\
\hline \multirow{4}{*}{ Boraginaceae } & 91. & $\begin{array}{l}\text { Buglossoides arvensis (L.) I.M. } \\
\text { Johnst. }\end{array}$ & + & + & Th & $\mathrm{L}$ & Jan-Apr \\
\hline & 92. & Cynoglossum lanceolatum Forssk. & + & + & $\mathrm{H}$ & $\mathrm{N}$ & Jun-Sep \\
\hline & 93. & Onosma thomsonii Clarke & + & - & $\mathrm{H}$ & $\mathrm{Mi}$ & Apr-May \\
\hline & 94. & Trichodesma indicum (L.) Lehm. & + & + & Th & $\mathrm{N}$ & Mar-Aug \\
\hline \multirow{3}{*}{ Brassicaceae } & 95. & Capsella bursa-pastoris (L.) Medik & - & + & Th & $\mathrm{L}$ & Feb-Jun \\
\hline & 96. & Lepidium sativum $\mathrm{L}$. & - & + & $\mathrm{H}$ & $\mathrm{N}$ & Apr-Jun \\
\hline & 97. & $\begin{array}{l}\text { Neslia apiculata Fisch., C.A. Mey. } \\
\& \text { Ave'-Lall. }\end{array}$ & - & + & Th & $\mathrm{L}$ & Mar-Apr \\
\hline Campanulaceae & 98. & Campanula pallida Wall. & + & - & $\mathrm{Th}$ & $\mathrm{N}$ & Apr-Jul \\
\hline Caprifoliaceae & 99. & $\begin{array}{l}\text { Lonicera quinquelocularis } \\
\text { Hardwicke }\end{array}$ & + & + & NP & $\mathrm{Me}$ & Mar-Jul \\
\hline Caryophyllaceae & 100. & $\begin{array}{l}\text { Arenaria neelgherrensis Wight \& } \\
\text { Arn. }\end{array}$ & + & - & Th & $\mathrm{L}$ & Mar-Aug \\
\hline
\end{tabular}




\begin{tabular}{|c|c|c|c|c|c|c|c|}
\hline & 101. & Silene conoidea L. & - & + & Th & $\mathrm{N}$ & Mar-May \\
\hline & 102. & Stellaria media (L.)Vill. & - & + & Th & $\mathrm{L}$ & Jan-Apr \\
\hline Celastraceae & 103. & $\begin{array}{l}\text { Maytenus royleana (Wall. ex M.A. } \\
\text { Lawson) Cufod. }\end{array}$ & + & + & NP & $\mathrm{N}$ & Feb-Apr \\
\hline \multirow{4}{*}{ Convolvulaceae } & 104. & Evolvulus alsinoides (L.) L. & + & - & $\mathrm{Ch}$ & $\mathrm{N}$ & Feb-Oct \\
\hline & 105. & Ipomoea eriocarpa $\mathrm{R} . \mathrm{Br}$. & + & - & $\mathrm{L}$ & $\mathrm{Mi}$ & Jul-Oct \\
\hline & 106. & Ipomoea hederacea Jacq. & + & - & $\mathrm{L}$ & $\mathrm{Mi}$ & Sep-Oct \\
\hline & 107. & Ipomoea pestigridis $\mathrm{L}$. & + & - & $\mathrm{L}$ & $\mathrm{Mi}$ & Aug-Oct \\
\hline Elaeagnaceae & 108. & $\begin{array}{l}\text { Elaeagnus parvifolia Wall. ex } \\
\text { Royle }\end{array}$ & + & + & NP & Mi & May-Jun \\
\hline \multirow{7}{*}{ Euphorbiaceae } & 109. & $\begin{array}{l}\text { Mallotus philippensis (Lamk.) Mull. } \\
\text { Arg. }\end{array}$ & + & + & MP & $\mathrm{Me}$ & Mar-May \\
\hline & 110. & Euphorbia esula L. & + & + & $\mathrm{H}$ & $\mathrm{N}$ & May-Aug \\
\hline & 111. & Euphorbia helioscopia L. & - & + & Th & $\mathrm{N}$ & Jan-Apr \\
\hline & 112. & Euphorbia hirta L. & + & + & Th & $\mathrm{N}$ & Jun-Dec \\
\hline & 113. & Euphorbia indica Lam. & + & + & Th & $\mathrm{N}$ & Jun-Sep \\
\hline & 114. & $\begin{array}{l}\text { Euphorbia prolifera Buch.-Ham. ex } \\
\text { D. Don }\end{array}$ & + & - & $\mathrm{H}$ & $\mathrm{L}$ & Mar-Jun \\
\hline & 115. & Euphorbia prostrata Ait. & + & - & $\mathrm{H}$ & $\mathrm{L}$ & Mar-Dec \\
\hline \multirow{15}{*}{ Fabaceae } & 116. & Acacia modesta Wall. & + & + & MP & $\mathrm{L}$ & Mar-May \\
\hline & 117. & Alysicarpus bupleurifolius (L.) DC. & + & - & Th & $\mathrm{N}$ & Sep-Oct \\
\hline & 118. & Argyrolobium roseum Jaub. & + & + & Th & $\mathrm{N}$ & Apr-Aug \\
\hline & 119. & Astragalus psilocentros Fisch. & + & + & NP & $\mathrm{L}$ & Mar-May \\
\hline & 120. & $\begin{array}{l}\text { Astragalus leucocephalus Grah.ex } \\
\text { Benth. }\end{array}$ & - & + & $\mathrm{Ch}$ & $\mathrm{L}$ & Mar-Jul \\
\hline & 121. & Butea monosperma (Lam.) Taubert & + & + & MP & $\mathrm{Me}$ & Mar-May \\
\hline & 122. & $\begin{array}{l}\text { Indigofera heterantha Wall. ex } \\
\text { Brandis }\end{array}$ & + & + & NP & $\mathrm{L}$ & Apr-May \\
\hline & 123. & Indigofera linifolia (L.f.) Retz. & + & - & $\mathrm{Ch}$ & $\mathrm{L}$ & May-Aug \\
\hline & 124. & $\begin{array}{l}\text { Lespedeza juncea var. sericea F.B. } \\
\text { Forbes \& Hemsl. }\end{array}$ & + & + & Th & $\mathrm{L}$ & Jun-Oct \\
\hline & 125. & Rhynchosia pseudo-cajan Camb. & + & + & NP & $\mathrm{N}$ & May-Jun \\
\hline & 126. & Lotus corniculatus L. & + & - & $\mathrm{H}$ & $\mathrm{L}$ & Mar-Apr \\
\hline & 127. & Medicago polymorpha L. & + & + & Th & $\mathrm{N}$ & $\begin{array}{l}\text { Round the } \\
\text { year }\end{array}$ \\
\hline & 128. & Melilotus indica (L.) All. & + & + & Th & $\mathrm{L}$ & Mar-May \\
\hline & 129. & Trifolium repens $\mathrm{L}$. & + & - & $\mathrm{H}$ & $\mathrm{N}$ & Mar-Apr \\
\hline & 130. & Vicia sativa $\mathrm{L}$. & - & + & $\mathrm{L}$ & $\mathrm{L}$ & May-Aug \\
\hline Fagaceae & 131. & Quercus incana W. Bartram & + & + & MP & $\mathrm{Me}$ & Apr-May \\
\hline Flacourtiaceae & 132. & Flacourtia indica (Burm. f.) Merill. & + & + & MP & $\mathrm{Mi}$ & Mar-Apr \\
\hline \multirow[t]{2}{*}{ Gentianaceae } & 133. & $\begin{array}{l}\text { Gentianodes decemfida (Ham.) } \\
\text { Omer, Ali \& Qaiser }\end{array}$ & - & + & $\mathrm{H}$ & $\mathrm{N}$ & Feb-Mar \\
\hline & 134. & Swertia petiolata D. Don & + & - & Th & $\mathrm{Mi}$ & Jul-Nov \\
\hline \multirow{4}{*}{ Geraniaceae } & 135. & $\begin{array}{l}\text { Erodium cicutarium (L.) L'Hér. ex } \\
\text { Aiton }\end{array}$ & - & + & $\mathrm{Th}$ & $\mathrm{L}$ & Mar-Apr \\
\hline & 136. & Geranium nepalense Sweet & + & + & $\mathrm{H}$ & $\mathrm{Mi}$ & Apr-Sep \\
\hline & 137. & Geranium ocellatum Camb. & - & + & $\mathrm{Th}$ & $\mathrm{Mi}$ & Mar-Apr \\
\hline & 138. & Geranium rotundifolium L. & + & + & Th & $\mathrm{N}$ & Mar-Apr \\
\hline \multirow{2}{*}{ Hypericaceae } & 139. & Hypericum oblongifolium Choisy & + & + & NP & $\mathrm{Mi}$ & Mar-Aug \\
\hline & 140. & Hypericum perforatum $\mathrm{L}$. & + & - & $\mathrm{Ch}$ & $\mathrm{N}$ & Jun-Sep \\
\hline \multirow{4}{*}{ Lamiaceae } & 141. & Ajuga bracteosa Wallich ex. Benth. & + & + & Th & $\mathrm{Mi}$ & Mar-Dec \\
\hline & 142. & Ajuga parviflora Benth. & + & + & Th & $\mathrm{Mi}$ & Mar-Jun \\
\hline & 143. & Anisomeles indica (L.) Kuntze & + & - & $\mathrm{H}$ & $\mathrm{Mi}$ & Apr-Sep \\
\hline & 144. & Calamintha umbrosa (M. Bieb.) & + & - & $\mathrm{H}$ & $\mathrm{N}$ & Apr-Jul \\
\hline
\end{tabular}


Amjad et al.

\begin{tabular}{|c|c|c|c|c|c|c|c|}
\hline & 145. & Fisch. \& C.A. Mey. & & & & & \\
\hline & 146. & Colebrookea oppositifolia Smith. & + & + & NP & $\mathrm{Me}$ & Jan-Apr \\
\hline & 147. & $\begin{array}{l}\text { Isodon rugosus (Wall. ex Benth.) } \\
\text { Codd }\end{array}$ & + & + & NP & $\mathrm{L}$ & Mar-Oct \\
\hline & 148. & $\begin{array}{l}\text { Micromeria biflora (Buch.-Ham.ex } \\
\text { D.Don) Benth. }\end{array}$ & + & + & Th & $\mathrm{L}$ & Apr-Oct \\
\hline & 149. & Otostegia limbata (Bth.) Bioss. & + & + & NP & $\mathrm{L}$ & Apr-Aug \\
\hline & 150. & Origanum vulgare $\mathrm{L}$. & + & + & $\mathrm{H}$ & $\mathrm{N}$ & Jul-Oct \\
\hline & 151. & Salvia moocroftiana Wall. ex Benth. & - & + & $\mathrm{Ch}$ & $\mathrm{Me}$ & Apr-Jun \\
\hline & 152. & Salvia plebeia $\mathrm{R} . \mathrm{Br}$. & + & - & $\mathrm{Ch}$ & $\mathrm{Mi}$ & Mar-Jun \\
\hline Loranthaceae & 153. & Loranthus pulverulentus Wall. & + & + & $\mathrm{NP}$ & $\mathrm{Me}$ & Dec-Apr \\
\hline \multirow{2}{*}{ Lythraceae } & 154. & Punica granatum $\mathrm{L}$. & + & + & NP & $\mathrm{N}$ & Apr-Jul \\
\hline & 155. & Woodfordia fruticosa (L.) Kurz & + & + & NP & $\mathrm{N}$ & Mar-May \\
\hline \multirow{5}{*}{ Malvaceae } & 156. & Malva parviflora $\mathrm{L}$. & + & - & Th & $\mathrm{Mi}$ & Dec-Mar \\
\hline & 157. & $\begin{array}{l}\text { Malvastrum coromandelianum (L.) } \\
\text { Garcke }\end{array}$ & + & + & Th & $\mathrm{N}$ & Mar-Sep \\
\hline & 158. & $\begin{array}{l}\text { Sida cordata (Burm.f.) Borss. var. } \\
\text { cordata }\end{array}$ & - & + & $\mathrm{Th}$ & $\mathrm{L}$ & $\begin{array}{l}\text { Round the } \\
\text { year }\end{array}$ \\
\hline & 159. & Sida cordifolia $\mathrm{L}$. & + & + & Th & $\mathrm{N}$ & Apr-Aug \\
\hline & 160. & Triumfetta pentandra A.Rich. & + & - & Th & $\mathrm{Mi}$ & Jul-Sep \\
\hline Menispermaceae & 161. & $\begin{array}{l}\text { Cissampelos pareira var. hirsuta } \\
\text { (Buch.-Ham. ex DC.) Forman }\end{array}$ & + & - & $\mathrm{L}$ & Mi & Jun-Sep \\
\hline Nyctaginaceae & 162. & $\begin{array}{l}\text { Boerhavia procumbens Banks ex } \\
\text { Roxb. }\end{array}$ & + & + & $\mathrm{H}$ & $\mathrm{N}$ & $\begin{array}{l}\text { Round the } \\
\text { year }\end{array}$ \\
\hline \multirow{2}{*}{ Oleaceae } & 163. & Olea ferruginea Royle & + & + & MP & $\mathrm{N}$ & Apr-May \\
\hline & 164. & Jasminum officinale L. & + & + & $\mathrm{L}$ & $\mathrm{N}$ & May-Jul \\
\hline Onagraceae & 165. & Oenothera rosea L.Herit.ex Ait. & + & + & $\mathrm{H}$ & $\mathrm{N}$ & Apr-Sep \\
\hline Oxalidaceae & 166. & Oxalis corniculata $\mathrm{L}$. & + & + & $\mathrm{H}$ & $\mathrm{N}$ & Mar-Dec \\
\hline Papaveraceae & 167. & Fumaria indica Pugsley & - & + & Th & $\mathrm{N}$ & Feb-Jun \\
\hline Phyllanthaceae & 168. & Phyllanthus urinaria L. & + & - & Th & $\mathrm{L}$ & Aug-Sep \\
\hline Plantaginaceae & 169. & Plantago lanceolata $\mathrm{L}$. & + & + & $\mathrm{H}$ & $\mathrm{N}$ & Mar-Aug \\
\hline Polygalaceae & 170. & $\begin{array}{l}\text { Polygala abyssinica R. Br. ex } \\
\text { Fresen. }\end{array}$ & + & - & $\mathrm{Th}$ & $\mathrm{Mi}$ & Apr-Sep \\
\hline \multirow{4}{*}{ Polygonaceae } & 171. & Polygonum aviculare L. & + & + & $\mathrm{H}$ & $\mathrm{L}$ & Mar-Sep \\
\hline & 172. & Polygonum plebeium $\mathrm{R}$. Br. & - & + & Th & $\mathrm{L}$ & Feb-Sep \\
\hline & 173. & Rumex dentatus $\mathrm{L}$. & - & + & $\mathrm{G}$ & $\mathrm{Me}$ & Mar-Apr \\
\hline & 174. & Rumex hastatus D.Don & - & + & $\mathrm{Ch}$ & $\mathrm{N}$ & Mar-Aug \\
\hline \multirow{3}{*}{ Primulaceae } & 175. & Anagallis arvensis $\mathrm{L}$. & - & + & Th & $\mathrm{N}$ & Feb-Mar \\
\hline & 176. & Androsace umbellata (Lour.) Merill & + & + & $\mathrm{H}$ & $\mathrm{N}$ & Mar-Apr \\
\hline & 177. & Myrsine africana $\mathrm{L}$. & + & + & NP & $\mathrm{N}$ & Mar-May \\
\hline \multirow{4}{*}{ Rananculaceae } & 178. & Clematis grata Wall. & + & + & $\mathrm{L}$ & $\mathrm{N}$ & Apr-Aug \\
\hline & 179. & Ranunculus laetus Wall. ex Royle & + & + & $\mathrm{H}$ & $\mathrm{Mi}$ & Apr-Aug \\
\hline & 180. & Ranunculus muricatus L. & - & + & Th & $\mathrm{Mi}$ & Mar-May \\
\hline & 181. & Thalictrum foliolosum DC. & + & - & $\mathrm{Ch}$ & $\mathrm{N}$ & Jul-Sep \\
\hline Rhamnaceae & 182. & $\begin{array}{l}\text { Ziziphus } \\
\text { mauritiana var. spontanea (Edgew.) } \\
\text { R.R. Stewart ex Qaiser \& Nazim. }\end{array}$ & + & + & MP & $\mathrm{N}$ & Jun-Sep \\
\hline \multirow{5}{*}{ Rosaceae } & 183. & $\begin{array}{l}\text { Duchesnea indica (Andrews) } \\
\text { Teschem. }\end{array}$ & + & + & $\mathrm{H}$ & $\mathrm{N}$ & Mar-May \\
\hline & 184. & Geum canadense Jacq. & + & + & $\mathrm{H}$ & $\mathrm{Mi}$ & Jun-Sep \\
\hline & 185. & Prunus persica (L) )Batsch & + & + & MP & $\mathrm{Mi}$ & Feb-Apr \\
\hline & 186. & Rosa brunonii Lindl. & + & + & $\mathrm{L}$ & $\mathrm{N}$ & Apr-Jun \\
\hline & 187. & Rubus fruticosus L. & + & + & NP & $\mathrm{Mi}$ & Apr-Oct \\
\hline Rubiaceae & 188. & Galium aparine $\mathrm{L}$. & + & + & Th & $\mathrm{L}$ & Mar-May \\
\hline
\end{tabular}




\begin{tabular}{|c|c|c|c|c|c|c|c|}
\hline & 189. & Rubia cordifolia $\mathrm{L}$. & + & + & $\mathrm{L}$ & $\mathrm{N}$ & Jun-Nov \\
\hline Rutaceae & 190. & Zanthoxylum armatum DC. & + & + & NP & $\mathrm{N}$ & Mar-Apr \\
\hline \multirow{2}{*}{ Salicaceae } & 191. & Casearia tomentosa Roxb. & + & + & MP & $\mathrm{Me}$ & Mar-Jun \\
\hline & 192. & Salix acomophylla Boiss. & + & + & MP & $\mathrm{Mi}$ & Oct-Mar \\
\hline Sapindaceae & 193. & Dodonaea viscosa (L.) & + & + & NP & $\mathrm{N}$ & Jan-Mar \\
\hline Saxifragaceae & 194. & Bergenia ciliata Sternb. & + & + & $\mathrm{H}$ & $\mathrm{Me}$ & Mar-Jul \\
\hline Scrophulariaceae & 195. & Verbascum thapsus $\mathrm{L}$. & - & + & Th & $\mathrm{Me}$ & Mar-Sep \\
\hline Simaroubaceae & 196. & Ailanthus altissima (Mill.) Swingle & + & + & NP & $\mathrm{Mi}$ & Jul-Aug \\
\hline \multirow{3}{*}{ Solanaceae } & 197. & Physalis divaricata D. Don & + & - & Th & $\mathrm{Mi}$ & Aug-Oct \\
\hline & 198. & Solanum nigrum L. & + & + & Th & Mi & $\begin{array}{l}\text { Round the } \\
\text { year }\end{array}$ \\
\hline & 199. & Solanum surattense Burm. f. & - & + & $\mathrm{H}$ & $\mathrm{Me}$ & Mar-Sep \\
\hline Urticaceae & 200. & $\begin{array}{l}\text { Debregeasia salicifolia (D. Don) } \\
\text { Rendle }\end{array}$ & + & + & NP & $\mathrm{Mi}$ & Mar-Jun \\
\hline Verbenaceae & 201. & Lantana camara $\mathrm{L}$. & + & + & NP & $\mathrm{Mi}$ & Mar-Oct \\
\hline Violaceae & 202. & Viola canescens Wall.ex Roxb. & + & + & $\mathrm{G}$ & $\mathrm{N}$ & Mar-Aug \\
\hline Vitaceae & 203. & Cissus carnosa Lam. & + & - & $\mathrm{L}$ & $\mathrm{Mi}$ & Jul-Sep \\
\hline
\end{tabular}

\section{Florisic composition}

\section{Florisic composition}

The flora of District Kotli consisted of 202 species of 71 families and 176 genera (Table 1). Of these, there were 159 species of dicotyledons, 36 species of monocotyledons, one species of gymnosperm and six species of pteridophytes. Pinus roxburghii was the only gymnosperm present in the investigated area. On the basis of growth form, 11 trees, 28 shrubs and 163 herbs comprised the $5.45 \%, 13.86 \%$ and $80.69 \%$ of the floral diversity of the investigated area (Table 2, Figure 2).
Asteraceae (23 Spp.) and Poaceae (20 Spp.) were the dominant families followed by Fabaceae (15 Spp.), Labiatae (11 Spp.), and Euphorbiaceae (7 Spp.). Acanthaceae, Amaranthaceae, Apiaceae, Cyperaceae, Malvaceae and Rosaceae had 5 species each. Boraginaceae, Convolvulaceae, Geraniaceae, Polygonaceae, Pteridaceae and Rananculaceae had 4 species each (Table 3, Figure 3). Each of the Apocynaceae, Brassicaceae, Caryophyllaceae, Primulaceae and Solanaceae had 3 species. The remaining 49 families had two or fewer species (Table 3).

Table 1. Taxonomic distribution of recorded plant species from District Kotli

\begin{tabular}{|l|l|l|l|l|l|}
\hline \multirow{2}{*}{ Taxa } & \multicolumn{3}{|c|}{ Taxonomic distribution } & \multirow{2}{*}{ Total } \\
\cline { 2 - 5 } & \multirow{2}{*}{ Pteridophytes } & \multirow{2}{*}{ Gymnosperms } & Angiosperms & Dicotyledons & \\
\cline { 3 - 6 } & & & Monocotyledons & . & \\
\hline Families & $3(4.23 \%)$ & $1(1.41 \%)$ & $11(15.49 \%)$ & $56(78.87 \%)$ & 71 \\
\hline Genera & $5(2.84 \%)$ & $1(0.57 \%)$ & $31(17.61 \%)$ & $139(78.98 \%)$ & 176 \\
\hline Species & $6(2.97 \%)$ & $1(0.50 \%)$ & $36(17.82 \%)$ & $159(78.71 \%)$ & 202 \\
\hline
\end{tabular}


Table 2. Summary of ecological characteristics of the flora of District Kotli

\begin{tabular}{|c|c|c|c|}
\hline S. No & Ecological Characteristics & No & Percentage \\
\hline \multicolumn{4}{|l|}{ Flora } \\
\hline I. & Total Species & 202 & - \\
\hline II. & Family & 71 & - \\
\hline III. & Genera & 176 & - \\
\hline \multicolumn{4}{|l|}{ Habit } \\
\hline I. & Tree & 11 & 5.45 \\
\hline II. & Shrub & 28 & 13.86 \\
\hline III. & Herb & 163 & 80.69 \\
\hline \multicolumn{4}{|c|}{ Seasonality/Aspect } \\
\hline IV. & Monsoon & 162 & 52.43 \\
\hline V. & Spring & 147 & 47.57 \\
\hline \multicolumn{4}{|c|}{ Life Form Spectra } \\
\hline I. & Megaphanerophyte & 11 & 5.45 \\
\hline II. & \begin{tabular}{|l|} 
Nanophanerophyte \\
\end{tabular} & 27 & 13.37 \\
\hline III. & Therophyte & 73 & 36.14 \\
\hline IV. & Hemicryptophyte & 39 & 19.31 \\
\hline V. & Geophyte & 21 & 10.40 \\
\hline VI. & Chamaephyte & 15 & 7.43 \\
\hline VII. & Lianas & 16 & 7.92 \\
\hline \multicolumn{4}{|c|}{ Leaf Spectra } \\
\hline I. & Leptophyll & 54 & 26.73 \\
\hline II. & Nanophyll & 73 & 36.14 \\
\hline III. & Microphyll & 53 & 26.24 \\
\hline IV. & Mesophyll & 22 & 10.89 \\
\hline
\end{tabular}

Table 3. Number of species in recorded plant families from District Kotli

\begin{tabular}{|l|l|l|l|l|l|}
\hline S.No & Family & $\begin{array}{l}\text { No. of } \\
\text { Species }\end{array}$ & S.No & Family & $\begin{array}{l}\text { No. of } \\
\text { Species }\end{array}$ \\
\hline 1. & Asteraceae & 23 & 37. & Berberidaceae & 01 \\
\hline 2. & Poaceae & 20 & 38. & Campanulaceae & 01 \\
\hline 3. & Fabaceae & 15 & 39. & Caprifoliaceae & 01 \\
\hline 4. & Labiatae & 11 & 40. & Celastraceae & 01 \\
\hline 5. & Euphorbiaceae & 07 & 41. & Colchicaceae & 01 \\
\hline 6. & Acanthaceae & 05 & 42. & Commelinaceae & 01 \\
\hline 7. & Amaranthaceae & 05 & 43. & Dryopteridaceae & 01 \\
\hline 8. & Apiaceae & 05 & 44. & Elaeagnaceae & 01 \\
\hline 9. & Cyperaceae & 05 & 45. & Fagaceae & 01 \\
\hline 10. & Malvaceae & 05 & 46. & Flacourtiaceae & 01 \\
\hline 11. & Rosaceae & 05 & 47. & Juncaceae & 01 \\
\hline 12. & Boraginaceae & 04 & 48. & Liliaceae & 01 \\
\hline 13. & Convolvulaceae & 04 & 49. & Loranthaceae & 01 \\
\hline 14. & Geraniaceae & 04 & 50. & Menispermaceae & 01 \\
\hline 15. & Polygonaceae & 04 & 51. & Nyctaginaceae & 01 \\
\hline
\end{tabular}




\begin{tabular}{|l|l|l|l|l|l|}
\hline 16. & Pteridaceae & 04 & 52. & Onagraceae & 01 \\
\hline 17. & Rananculaceae & 04 & 53. & Orchidaceae & 01 \\
\hline 18. & Apocynaceae & 03 & 54. & Oxalidaceae & 01 \\
\hline 19. & Brassicaceae & 03 & 55. & Papaveraceae & 01 \\
\hline 20. & Caryophyllaceae & 03 & 56. & Phyllanthaceae & 01 \\
\hline 21. & Primulaceae & 03 & 57. & Pinaceae & 01 \\
\hline 22. & Solanaceae & 03 & 58. & Plantaginaceae & 01 \\
\hline 23. & Asparagaceae & 02 & 59. & Polygalaceae & 01 \\
\hline 24. & Dioscoreaceae & 02 & 60. & Rhamnaceae & 01 \\
\hline 25. & Gentianaceae & 02 & 61. & Rutaceae & 01 \\
\hline 26. & Hypericaceae & 02 & 62. & Sapindaceae & 01 \\
\hline 27. & Lythraceae & 02 & 63. & Saxifragaceae & 01 \\
\hline 28. & Oleaceae & 02 & 64. & Scrophulariaceae & 01 \\
\hline 29. & Rubiaceae & 02 & 65. & Selaginellaceae & 01 \\
\hline 30. & Salicaceae & 02 & 66. & Simaroubaceae & 01 \\
\hline 31. & Adoxaceae & 01 & 67. & Smilacaceae & 01 \\
\hline 32. & Aizoaceae & 01 & 68. & Urticaceae & 01 \\
\hline 33. & Anacardiaceae & 01 & 69. & Verbenaceae & 01 \\
\hline 34. & Araceae & 01 & 70. & Violaceae & 01 \\
\hline 35. & Araliaceae & 01 & 71. & Vitaceae & 01 \\
\hline 36. & Balsaminaceae & 01 & & & \\
\hline
\end{tabular}

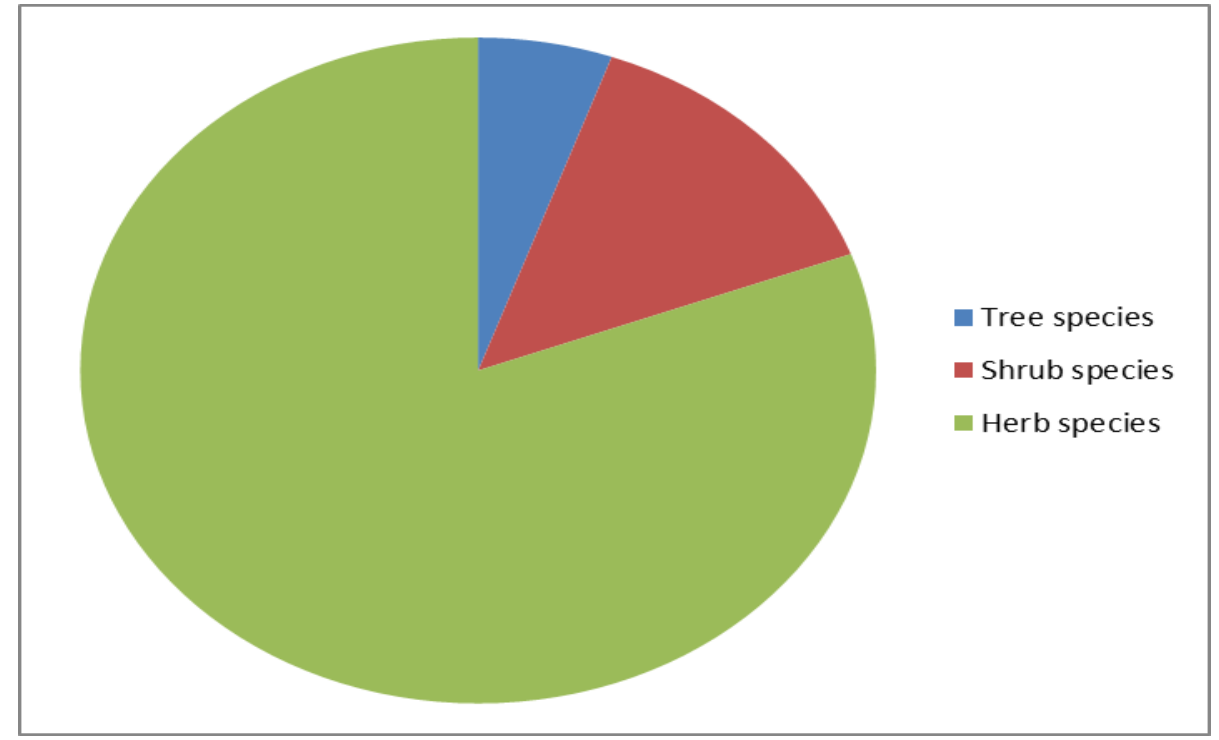

\section{Figure 2. Percent share of various habit forms of vegetation of the District Kotli}

Life form and its seasonal variation

The flora of the study area was overall dominated by therophytes (73 Spp., 36.14 $\%$ ), followed by hemicryptophytes (39 Spp., $19.31 \%)$, nanophanerophytes (27 Spp., 13.37\%), geophytes (21 Spp., 10.40\%), lianas (16 Spp., 7.92\%), chamaephytes (15 Spp., 7.43\%), and megaphanerophytes (11 Spp., 5.45\%) (Table 2).

Seasonal variation in life form was also reflected in the data. In spring there were $36.05 \%$ (53 Spp) therophytes, $21.09 \%$ (31 
Spp.) hemicryptophytes, $17.69 \%$ (26 Spp.) nanophanerophytes, $8.16 \% \quad(12 \quad$ Spp.) geophytes, $\quad 7.48 \% \quad(11 \quad$ Spp.) megaphanerophytes, $5.44 \%$ (8 Spp.) lianas, and $4.08 \%$ (6 Spp.) chamaephytes recorded. While in the monsoon season, there were 30.86\% (50 Spp) therophytes, 19.14\% (31 Spp.) hemicryptophytes, $16.67 \%$ (27 Spp.) nanophanerophytes, $11.11 \% \quad(18 \quad$ Spp. $)$ geophytes, 8.64\% (14 Spp.) lianas, 6.79\% (11 Spp.) megaphanerophytes, and 6.79\% (11 Spp.) chamaephytes recorded (Table 4 Figure 4). The percentage of therophytes was high during spring as compared to monsoon season.

Table 4. Seasonal variation in life form and leaf size spectra of plants of District Kotli

\begin{tabular}{|c|c|c|c|c|}
\hline \multirow[t]{3}{*}{ Parameters } & \multicolumn{4}{|c|}{ Season/Aspect } \\
\hline & \multicolumn{2}{|c|}{ Monsoon } & \multicolumn{2}{|c|}{ Spring } \\
\hline & No & $\%$ & No & $\%$ \\
\hline \multicolumn{5}{|l|}{ A. Life Form } \\
\hline Megaphanerophyte & 11 & 6.79 & 11 & 7.48 \\
\hline Nanophanerophyte & 27 & 16.67 & 26 & 17.69 \\
\hline Therophyte & 50 & 30.86 & 53 & 36.05 \\
\hline Hemicryptophyte & 31 & 19.14 & 31 & 21.09 \\
\hline Geophyte & 18 & 11.11 & 12 & 8.16 \\
\hline Chamaephyte & 11 & 6.79 & 06 & 4.08 \\
\hline Lianas & 14 & 8.64 & 08 & 5.44 \\
\hline Total & 162 & & 147 & \\
\hline \multicolumn{5}{|l|}{ B. Leaf Spectra } \\
\hline Leptophyll & 37 & 22.84 & 42 & 28.57 \\
\hline Nanophyll & 61 & 37.65 & 56 & 38.10 \\
\hline Microphyll & 46 & 28.40 & 33 & 22.45 \\
\hline Mesophyll & 18 & 11.11 & 16 & 10.88 \\
\hline Total & 162 & & 147 & \\
\hline
\end{tabular}




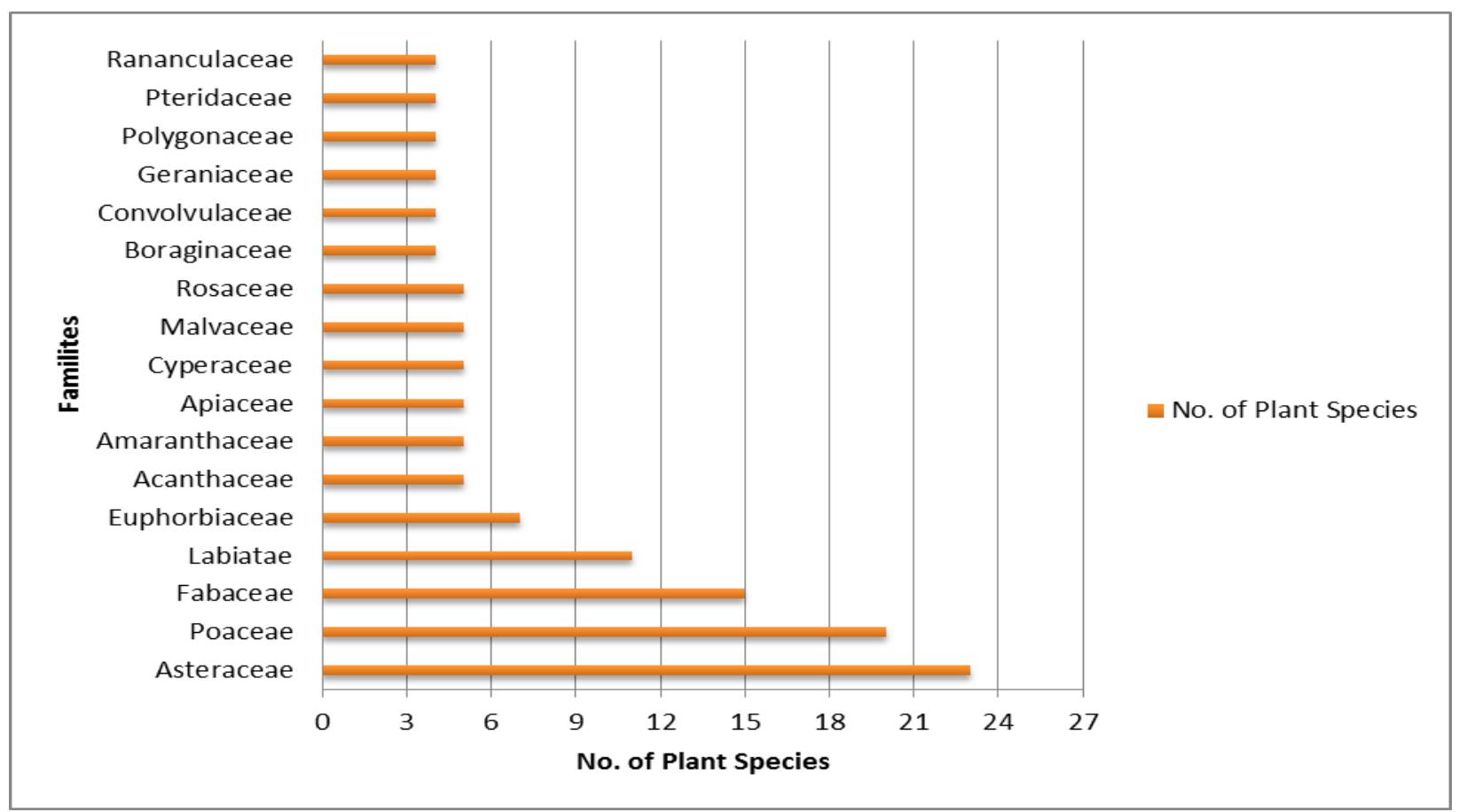

Figure 3. Top 17 plant families represented by highest number of species in the study area which share $64.36 \%$ of the total species

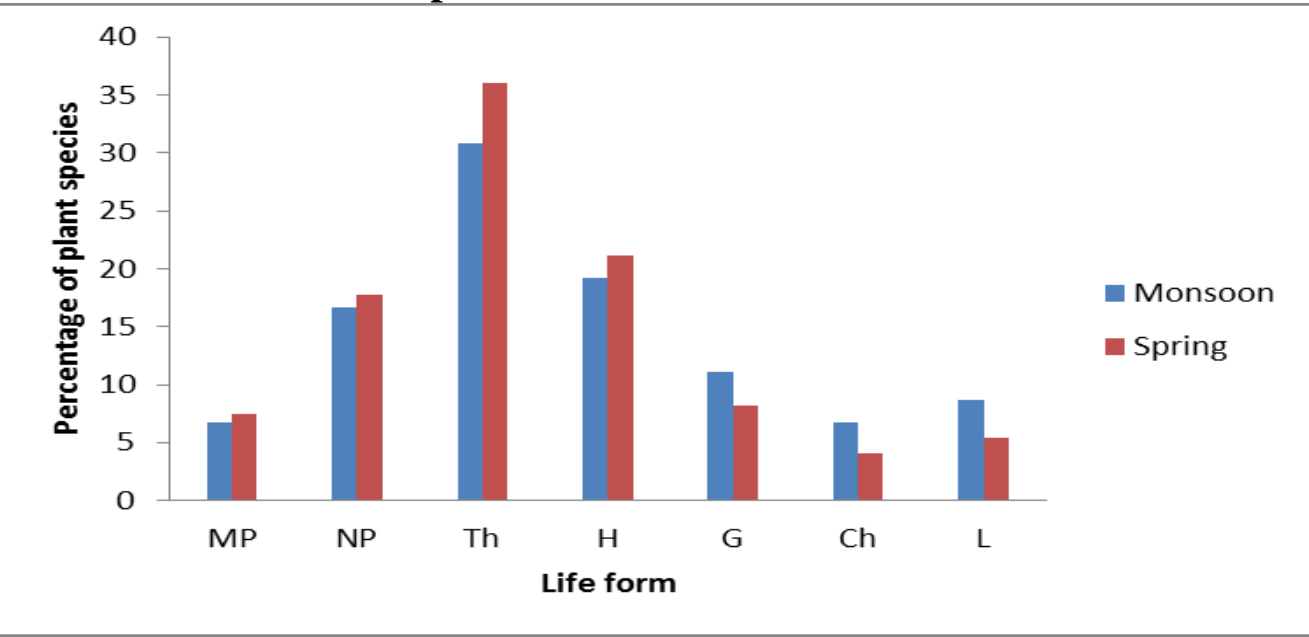

Figure 4. Graphical representation of seasonal variation in life form of District Kotli

Leaf spectra and its seasonal variation

The flora of study area was overall dominated by nanophylls (73 Spp; 36.14\%) followed by leptophylls (54 Spp.; 26.73\%) and microphylls (53 Spp.; 26.24\%). While mesophylls (22 Spp.; 10.89\%) were much lower in number (Table 2). Seasonal variation in leaf spectra shows that during the spring season there were $38.10 \%$ (56
Spp.) nanophylls, $28.57 \% \quad$ (42 Spp.) leptophylls, 22.45\% (33 Spp.) microphylls and $10.88 \%$ (16 Spp.) mesophylls. While in the monsoon season there were $37.65 \%$ (61 Spp.) nanophylls, $28.40 \%$ (46 Spp.) microphylls, $22.84 \%$ (37 Spp.) leptophylls and $11.11 \%$ (18 Spp.) mesophylls recorded (Table 4, Figure 5). 


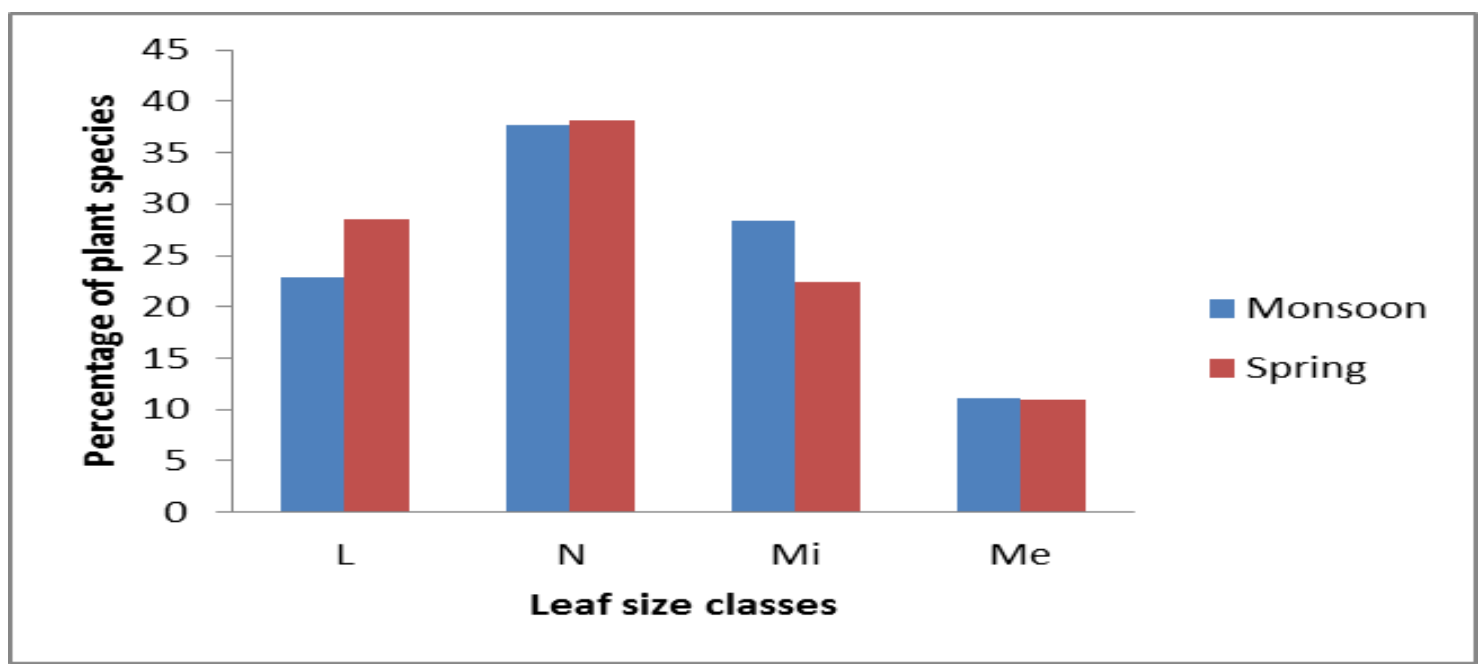

Figure 5. Graphical representation of seasonal variation in leaf spectra

\section{Phenological behaviour}

Four different flowering seasons could be observed in the investigated region (Figure 6 ). The first flowering season stretches from March to May (spring) during which 52.81 $\%$ of species were found in flowering. The second season (summer) stretches from June to August during which $53.80 \%$ of species were found in bloom. The third season (autumn) occurs from September to October during which $34.40 \%$ plants were in flower. The fourth season (winter) occurs from November to February during which only $10.40 \%$ species were found in flower.

The flowering trend decreased from spring into the winter season. It was observed that the majority of plant species (122 Spp.;
$60.40 \%$ ) were found in flower during April followed by May (111 Spp.; 54.95\%) and July (111 Spp.; 54.95\%), and then August (108 Spp.; 53.47\%). Whereas the least number of species were found in flower during the months of December (17 Spp. $8.42 \%$ ) and January (18 Spp.; 8.91\%). The maximum number of plant species initiated flowering during the month of March (60 spp. 29.70\%) followed by April (43 Spp.: 21.29\%), June (25 Spp. 12.38\%) and July (23 Spp.; 11.39\%. (Table 5, Figure 7). Peak flowering in trees, shrubs and herbs occurred during April, while among the grasses flowering was at a peak during August. Ferns for the most part reproduced during June-August.

Table 5. Average monthly phenological findings for the flora of Kotli, AJK

\begin{tabular}{|l|l|l|l|l|}
\hline \multirow{2}{*}{ Months } & \multicolumn{3}{|l|}{ Species found in flowering } & \multicolumn{2}{l|}{ Species started flowering } \\
\cline { 2 - 5 } & No & $\%$ & No & $\%$ \\
\hline January & 18 & 8.91 & 12 & 5.94 \\
\hline February & 28 & 13.86 & 11 & 5.45 \\
\hline March & 87 & 43.07 & 60 & 29.70 \\
\hline April & 122 & 60.40 & 43 & 21.29 \\
\hline May & 111 & 54.95 & 10 & 4.95 \\
\hline June & 107 & 52.97 & 25 & 12.38 \\
\hline July & 111 & 54.95 & 23 & 11.39 \\
\hline August & 108 & 53.47 & 07 & 3.47 \\
\hline September & 89 & 44.06 & 06 & 2.97 \\
\hline October & 50 & 24.75 & 01 & 0.50 \\
\hline November & 21 & 10.40 & 01 & 0.50 \\
\hline December & 17 & 8.42 & 03 & 1.49 \\
\hline
\end{tabular}




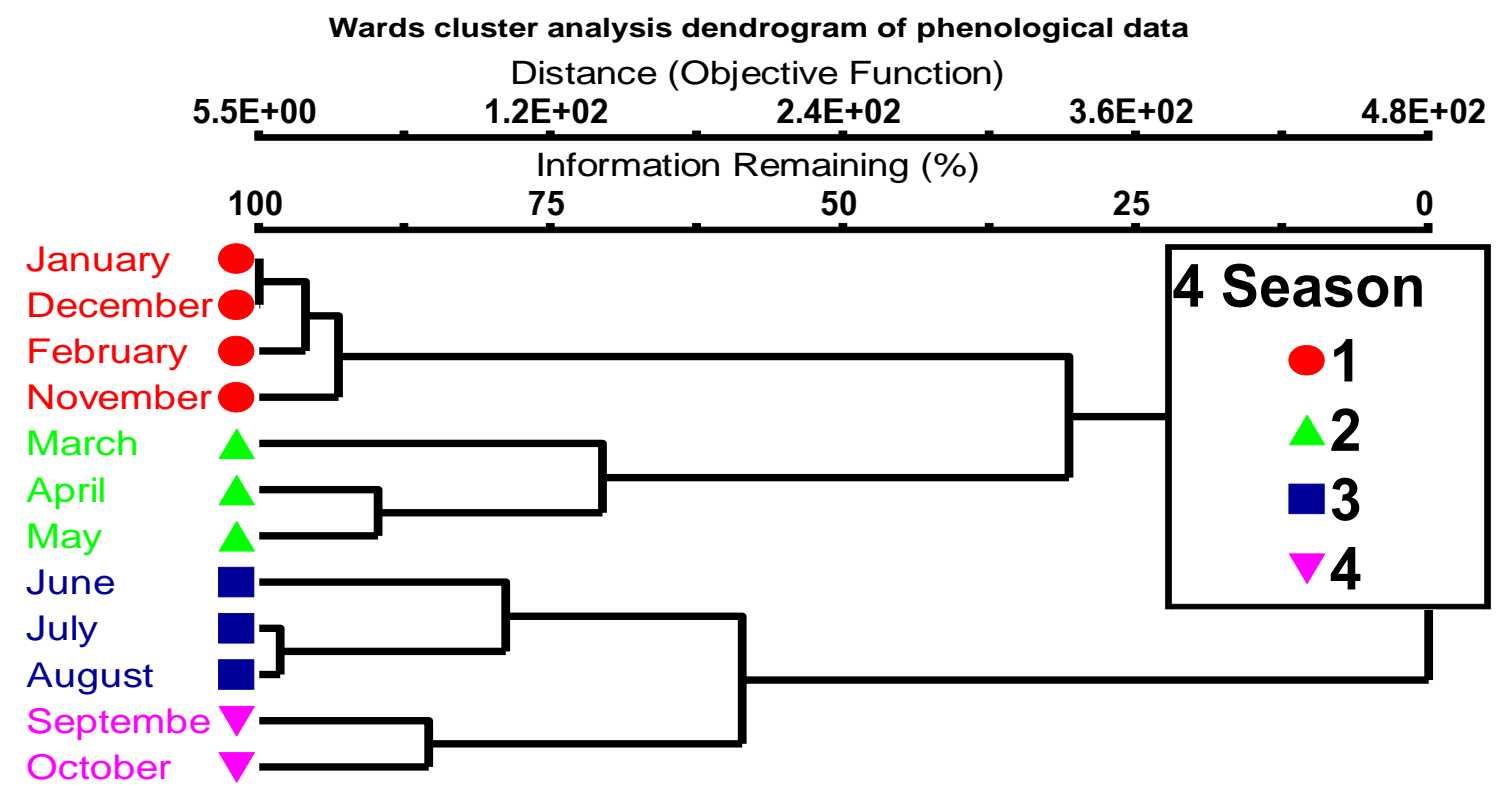

Figure 6. Wards cluster analysis dendrogram based on the phenological pattern of the vascular flora of Kotli District, AJ\&K

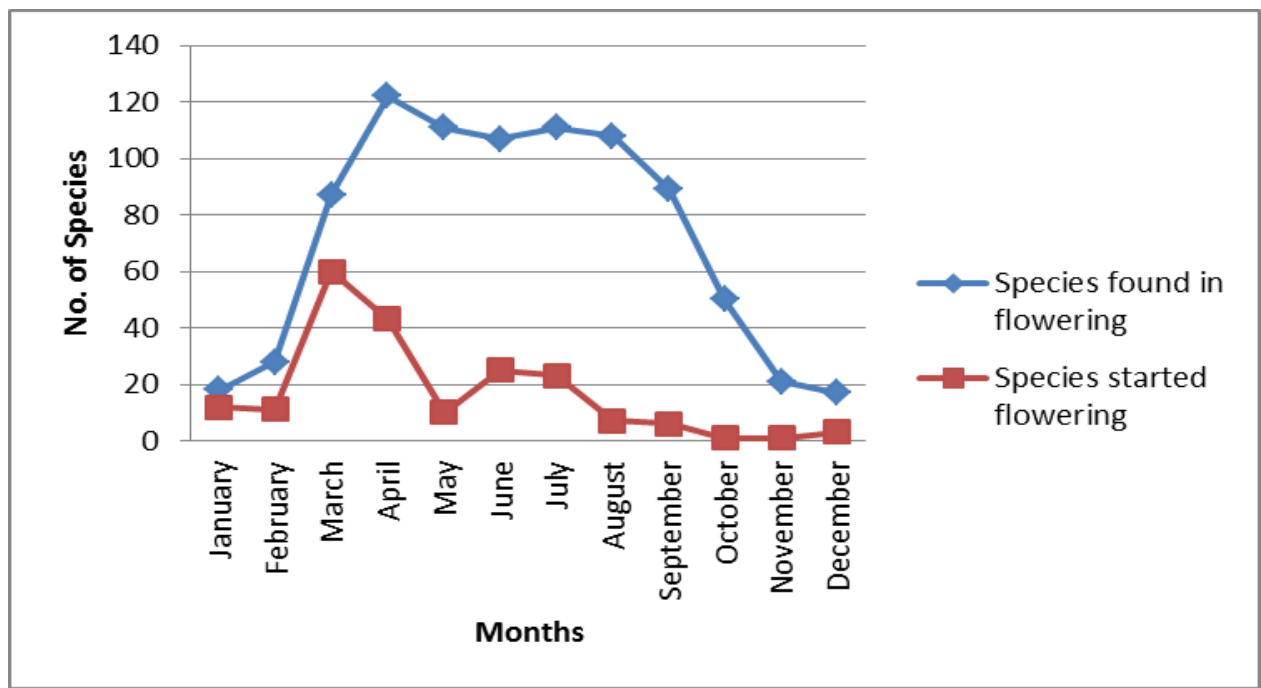

Figure 7. Seasonal variation in phenological pattern of vascular flora of Kotli District, AJK

\section{Discussion}

Floristic composition

Floristic diversity is the total number of plant species of an area and can be affected by anthropogenic interactions, grazing, soil erosion and natural disasters such as earthquakes that may cause landslides. The floristic check list of District Kotli is composed of 202 species that were distributed into 71 families and 176 genera. Asteraceae, Poaceae, Fabaceae, Labiatae are the leading families in the area on the basis of the number of species, but on the basis of importance value, Poaceae and Pinaceae are the dominant families both during surveys during the monsoon and the spring seasons. Stewart also reported these families to be well represented in Pakistan and Azad Kashmir [34], as did Ilys and his co-workers from Kablal vallyey of Swat [35], Tanvir and his coworkers from Bagh district of Azad Kashmir [36] and Amjad and his co- 
workers from Nikyal valley of Azad Kashmir [28]. Some other works also report similar findings in other regions of Pakistan $[1,15,27,37]$ and abroad [38, 39]. Our results are consistently supported by them as Poaceae and Asteraceae have emerged as the common families in the investigated area. Both these two families have also been reported to contain more species as compared to other families by Ali and his co-workers from the Chilas valley, Swat [16] and Hussain and Chaudhary from the Samhani valley, Azad Kashmir [40]. The members of the Poaceae and Asteraceae are found in a diverse range of habitats due to their broad ecological amplitude.

Many species have been floristically listed from District Kolti, however most of them have a limited distribution on a quantitative basis due to their restricted life cycle. High species number is the indication of high species diversity and richness in the area. Qualitative features like floristic composition alone cannot provide the true picture of subtropical zone of Kotli district therefore the quantitative parameters and ecological behavior of vegetation resources like habit, phenology, life from and leaf spectra and ethno ecological potential should also be studied for a full description of the plant resources of this area.

\section{Life form}

The biological spectrum of the vegetation can be developed by classifying all the species of higher plants in to life form classes and expressing their ratio both in numbers as well as percentages. The biological spectrum provides an indication of the existing climatic condition and can be helpful in comparing widely separated geographical communities. However the proportion of various life forms within the biological spectrum may be changed due to biotic influences such as deforestation, overgrazing, agricultural practices and trampling etc $[3,23,41]$.
The life form of the flora and different plant communities in the study area indicates that therophytes are dominant followed by hemicryptophytes both in the spring and monsoon seasons. Geophytes, nanophanerophyte, lianas, chamaephytes and megaphanerophytes are the next most important on the basis of the number of species. Therophytes are indicators of subtropical and desert climates while hemicryptophytes are more characteristic of cool and humid conditions $[18,23,28,42$, 43]. The reported life forms reflects the climatic conditions in the study area with changes occuring from the dry subtropical zone to the humid zone with increasing altitude. These results are in line with Sher and his co-worker who reported the dominance of therophytes in subtropical forest in Chagharzai Bunir [44]. Similarly Ali and his coworker reported the dominance of therophytes from the Chail valley in Swat which is also consistent with our findings [16].

The dominance of therophytes over other life forms in the study area can be interpreted as not only a response to the harsh climate but also to anthropogenic pressures on the flora. The other possible reason could be the availability of moisture in the form of rain. Qureshi and his coworker also reported similar findings from Koont [45, 46]. Our results are also consistent with Costa and his coworkers who reported the dominance of therophytes in south eastern Brazil [47]. Our findings are also supported by Kar and his coworkers who reported the dominance of therophytes from grassland in Odisha, India [48]. However, the dominance of therophytes in almost all zones might also be due to habitat disturbance, deforestation, overgrazing and trampling [44, 49-51].

The percentage of therophytes was high in spring as compared to the monsoon season because in spring season there is always a 
flush of annual plants [18]. Hussain and his co-workers reported that in Girbanr and Dabargai Hills therophytes were dominant due to unfavorable habitat conditions [52]. Most researchers reported similar conclusions $[48,53,28]$ from certain other parts of world and our findings in this regard are supported by them.

Kotli is warm at lower altitudes and climatically cool at higher altitudes; this was reflected in the number of hemicryptophytes which increased in the sub-tropical broad leaf humid forest association (1550m$1897 \mathrm{~m})$. Saxena and his coworkers considered hemicryptophytes as the dominant life form in temperate or humid conditions and support our results [54].

The final life form class, the phanerophytes, decreased with altitude in the study area. Phanerophytes are best represented as a class in open physiognomies [55]. The climate of the Kotli District is suitable to support the growth of phanerophytes but severe anthropogenic pressures have greatly decreased their dominance in the study area.

\section{Leaf spectra}

The leaf size spectrum is helpful in understanding the physiological processes of plant species. The current study reveals the dominance of nanophylls followed by leptophylls in the study area. Badshah and his coworkers reported nanophylly as the dominant leaf size in Waziristan [21]. Similarly Sher \& Khan reported high percentages of leptophylls and nanophylls from the Chagarzai area [44]. Our findings are thus in line with these previous studies. Malik and his co-workers reported the dominance of microphylls in other areas of Azad Jammu and Kashmir [18, 23]. The disagreement with their results is due to differences in climatic conditions and altitude. The leaf size along with the habit and root system can be helpful in determining the climatic zone of a study area. Small sized leaves are an indicator of dry and xeric conditions [18, 23, 25, 28]. This is because in dry areas and particularly in mountains, the soil has a low nutrient and water content making it difficult for plant roots to absorb moisture and nutrient and thus encouraging the dominance of nanophyllous and leptophyllous vegetation, as was observed in our study area. The ratio of different leaf size classes changes during different seasons in the study area due to the presence of annuals and rhizomatous geophytes. Malik and his coworkers and Batalha and Martins also reported that leaf size is directly related to the soil conditions and the availability of moisture [18, 23, 55]. Leaf size alone could not be used to identify the climate or specific zone of the investigated area; root system and the habit of plants might play an equal role.

\section{Phenology}

According to the International Biological Program (IBP), phenology refers to "the study of the timing and causes of repeated biological phenomena with respect to nonliving and living factors and the relationship between the phases of different or the same species" $[9,10]$. Small variations in climate can have a great effect on vegetation [11], while vegetation can be characterized by different patterns of phenological events [12].

Flowering can be induced by a change in a variety of climatic factors such as significant rain in the months of winter and summer, temperature differences, changes in photoperiodism, and drought both at community or species level $[56,57]$. In the investigated area, the majority of plant species initiated flowering at the end of March which may be due to the onset of warmer weather [58]. Flower initiation and development and other growth activities are generally at their peak during rainy and early spring season in Caatinga [59], which is in line with our findings. 
In the investigated area, the peak time for flowering was April-September. Less flowering was observed from November to February. Kakim and Yadava [60] similarly reported that in the sub-tropical forests of Manipur India flowering occurred during the months of April to September, while Jadeja and Nakkar [11] reported that in Gujrat India maximum flowering occurred in the months of January and February. This difference might be due to climatic effects and differences in altitude and latitude. Our results are also in contrast with Malik and Malik who reported that maximum flowering occurred during July and August in the Ganga Chotti and Beddori hills of Azad Kashmir, which again may be due to a higher altitude, rainfall and saturation in their study area [61].

With a change in climatic conditions, the flowering period of plant species can be affected. A change in temperature can cause a shift in the reproductive phenology of plant species [62]. An increase in temperature advances the phenology of many plant species [63]. In the investigated area many herbaceous species were in flowering condition in the month of March when there was a slight increase in temperature otherwise flowering occurred in the month of April. So our findings are in accordance with previous reports.

Nautiyal and his coworkers reported that flowering in many plant species of the alpine zone occurred after snow fall [64]. In the investigated area flowering started in the month of March for the majority of the woody plant species so our findings in this regard are in accordance with this study. The majority of tree species were in flower during April which is also in accordance with Dar and Malik, who reported maximum flowering among the tree species during April while working in Lawat district, Azad Kashmir [65].
Golluscio and his coworkers observed that grasses, members of Poaceace, had the majority of their phenological activities during the autumn and winter seasons as compared to the non-grass plant species which had different starting and ending dates of vegetative and reproductive growth [66]. In our case too most of the grasses bloomed in autumn and early winter so our finding is in line with this.

Changes in climatic factors such as temperature and humidity can produce changes in the phenology of the plant species. Similarly urbanization is a major factor of global climatic change. There was an uneven fluctuation in temperature throughout the year in urban areas of Canada which has direct impact on vegetation phenology [67]. Similarly in our case, rise in temperature from March to May favored the flowering of plants and further increase in temperature caused onset and maturation of fruits from June - September. Biotic factors can also have a profound influence on the plant phenological events [68]. This has been observed in our case as temperatures generally decreased during October and November to promote leaf fall and in June and July the grazing pressure increased which facilitated the second dormant phase. The grazing of annual herbaceous plant species prior to flowering will restrict the population because in this case seeds will be the only propagation source for regeneration and survival. Therefore it is essential to coordinate the grazing period with the flowering cycle in order to promote sustainable use of plant resources.

\section{Conclusion}

The district Kotli is rich in biodiversity. The high percentage of therophyte and nanophyllous species are due to the subtropical climate of area. The high proportion of therophyte also reflect that area is under sever biotic pressure due to 
deforestation and overgrazing Majority of species are becoming endangered in the area, therefore immediate conservations measures are needed to preserve the endangered flora. The grazing of annual herbaceous plant species prior to flowering will restrict the population because in this case seeds will be the only propagation source for regeneration and survival. Therefore it is essential to coordinate the grazing period with the flowering cycle in order to promote sustainable use of plant resources.

\section{Authors' contributions}

Conceived and designed the experiments: M Arshad, R Qureshi \& SN Mirza, Methodology: MS Amjad \& M Arshad, Performed the experiments: MS Amjad, Analyzed the data: MS Amjed \& S Page, Contributed reagents/ materials/ analysis tools: MS Amjed \& M Arshad, Wrote the paper: MS Amjad \& S Page.

\section{Acknowledgements}

This is the part of PhD thesis of First Author. The authors are thankful to the IDEA WILD USA and HEC, Pakistan for providing funding to conduct this research work under his International Research Supportive Initiative Programme (IRSIP). We are also thankful to Dr. Muhammad Ilyas and Dr. Mushtaq Ahmad for the taxonomical assistance.

\section{References}

1. Hussain F, Sha SM, Badshah L \& Durrani MJ (2015). Diversity and ecological characteristics of flora of Mastuj valley, district Chitral, Hindukush range, Pakistan. Pak J Bot 47(2): 495-510.

2. Ali SI (2008). The significance of flora with special reference to Pakistan. Pak J Bot 40(30): 967-971.

3. Badshah L, Hussain F \& Sher Z (2013). Floristic inventory, ecological characteristics and biological spectrum of rangeland, District Tank, Pakistan. Pak J Bot 45(4): 1159-1168.
4. Cain SA (1950). Life form and phytoclimates. Bot Rev 16: 1-32.

5. Mera AG, Hagen MA \& Orellana JAV (1999). Aerophyte, a new life form in Raunkiaer's classification. Journal of Vegetation Science 10: 65-68.

6. Raunkiær C (1934). The life forms of plants and statistical plants geography being the collected papers of C. Raunkiaer. Clarend on press, Oxford.

7. Mueller-Dombois D \& Ellenberg H (1974). Aims and Methods of Vegetation Ecology. John Wiley and Sons Inc 1974.

8. Oosting HJ (1956). The Study of Plant Communities. $2^{\text {nd }}$ Ed. W. H. Freeman and Co., Sanfransisco $11 \mathrm{p}$.

9. Lieth H (1974). Phenology and Seasonality Modeling. Springer, New York. 444 p.

10. Richardson AD, Keenan TF, Migliavacca M, Ryu Y, Sonnentag O \& Toomey M (2013). Climate change, phenology, and phenological control of vegetation feedbacks to the climate system. Agricultural and Forest Meteorology 169: 156-173.

11. Jadeja BA \& Nakar RN (2010). Phenological studies of some tree species from Girnar reserve forest Gujarat India. Plant archives 10(2): 825 $-828$.

12. Opler PA, Frankie GW. \& Baker HG (1980). Comparative phenological studies of shrubs and tree lets in wet and dry forests in the low lands of Costa Rica; J Ecol 68: 167-186.

13. Borchert R, Rivera G \& Hagnauer W (2002). Modification of vegetative phenology in a tropical semideciduous forest by abnormal drought and rain. Biotropica 34: 27-39.

14. Fox JF (1976). Constrains on the natural regeneration of tropical moist forest. For Ecol Manage 1: 37-65.

15. Samreen U, Ibrar M \& Badshah L (2016). Floristic composition, Ecological characters and Biological characters of Darazinda, Pakistan. International Invention Journal of Agricultural and Soil Science 9-21. 
16. Ali A, Badshah L, Hussain F \& Shinwari ZK (2016). Floristic composition and ecological characteristics of plants of chail valley, district Swat, Pakistan. Pak $J$ Bot 48(3): 1013-1026.

17. Khan M, Hussain F \& Musharaf S (2014). Floristic Composition and Ecological Characteristics of Shahbaz Garhi, District Mardan. Pakistan Global Journal of Science Frontier Research Biological Science 14(1): 1-11.

18. Malik NZ, Malik ZH, Arshad M, Noshad Q \& Rashid N (2013). Life form and Leaf size Spectra reported in Moist Temperate forest of Pir-Chinassi Hills, District muzaffarabad Azad Jammu and Kashmir, T. J Agri Res 1(1): 1-7.

19. Hussain F, Shah SM \& Badshah L (2012). Floristic and vegetation diversity of some aquatic habitats of Mastuj Valley, Hindikush region, District Chitral, Pakistan. Pak J Pl Sci 18(1): 55-68.

20. Khan M, Hussain F, Musharaf S \& Imdadullah (2011). Floristic composition, life form and leaf size spectra of the coal mine area vegetation of Darra Adam Khel, Khyber Pakhtonkhwa, Pakistan. Journal of Biodiversity and Environmental Sciences 1(3): 1-6.

21. Badshah L, Hussain F \& Akhtar N (2010). Vegetation structure of subtropical forest of Tabai, South Waziristan, Pakistan. Front Agric China 4(2): 232 236.

22. Amjad MS, Qaeem Mf, Ahmad I, Khan SU, Chaudhari SK, Zahid Malik N, Shaheen H \& Khan AM (2017). Descriptive study of plant resources in the context of the ethnomedicinal relevance of indigenous flora: A case study from Toli Peer National Park, Azad Jammu and Kashmir, Pakistan. PLoS ONE 12(2): 1-31.

23. Malik NZ, Hussain F \& Malik ZH (2007). Life form and leaf size spectra of plant communities harbouring at Ganga Chotti and Bedorii hills" Int j Agri Bio 15(6): 833-838.
24. Ajaib M, Khan Z, Muhammad S \& Mahmood R (2008). Biological spectra of Saney Baney Hills district Kotli Azad Jammu and Kashmir," P J Sci 60 (1-2): 53-58.

25. Amjad MS (2012). Life form and leaf size spectra of vegetation in Kotli hills, Azad Jammu and Kashmir (Pakistan). Greener J Agric Sci 2(7): 345-50.

26. Nazir A, Malik RN \& Shaheen H (2014). Floristic composition, life form and leaf spectra of plant communities recorded at Sarsawa hills district Kotli. Afr J soil sci 2(4): 76-78.

27. Arshad MK, Khan M, Qureshi R, Qaseem MF, Munir M, Ilyas M. \& Saqib Z (2015). Foristic checklist of district Kotli, Azad Jammu \& Kashmir. Pak J Bot 47(5): 1957-1968.

28. Amjad MS, Arshad M, Sadaf HM, Shahwar D, Akrim F \& Arshad, A (2016). Floristic compositon, biological spectrum and conservation status of vegetation in Nikyal valley, Azad Jammu and Kashmir. Asi P J Trop Dis 6(1): 63-69.

29. Nasir E \& Ali SI (1970-1989). Flora ofPakistan, Islamabad, Karachi.

30. Ali SI \& Qaiser M (1993-2015). Flora of Pakistan Nos. 194-220. Karachi.

31. Hussain F (1989). Field and Laboratory Manual of Plant Ecology. UGC. Islamabad.

32. McCune B \& Grace JB (2002). Analysis of Ecological Communities. MjM Software, Gleneden Beach, Oregon, USA.

33. McCune B \& Mefford MJ (2005). Multivariate Analysis of Ecological Data (PCORD Version 5.10 Mj-M Software) Gleneden Beach, Oregon: United State of America.

34. Stewart RR (1972). An annotated catalogue of Vascular plants of WestPakistan and Kashmir. Karachi: Fakhri Printing Press.

35. Ilyas M, Qureshi R, Arshad M \& Mirza SN (2013). A preliminary chick list of the vascular Flora of Kabal Valley, 
Swat, Pakistan. Pak J Bot 45(2): 605615.

36. Tanvir M, Murtaza G, Ahmad KS \& Salman M (2014). Floral diversity of District Bagh, Azad Jammu and Kashmir Pakistan. Universal J Plant Sci 2(1): 1-13.

37. Sultan-Ud-Din, Ahmad H, Ali H \& Ali H (2016). Floristic composition and life form classes of district Shangla, Khyber Pakhtunkhwa, Pakistan. J Bio Env Sci 8(3): 187-206.

38. Muthuramkumar S, Ayyappan N, Parthasarathy N, Divya M, Raman STR, Arthur SM \& Arul PL (2006). Plant community structure in tropical rain forest fragments of the Western Ghats, India. Biotropica 38 (2): 143-160.

39. Mendez E (2005). The vegetation of the Laguna de Llancanelo Provincial Reserve (Mendoza, Argentina). Candollea 60 (1): 123-148.

40. Hussain T \& Chaudhary MI (2009). A Floristic Description of Flora and Ethnobotany of Samahni Valley (A.K.), Pakistan. Ethnobotanical Leaflets 13: 873-99.

41. Hussain F, Shah SM \& Badshah L (2012). Floristic and vegetation diversity of some aquatic habitats of Mastuj Valley, Hindikush region, District Chitral, Pakistan. Pak J Pl Sci 18(1): 55-68.

42. Cain SA \& Castro GM (1959). Manual of Vegetation analysis. Harper, NY. 325 p.

43. Shimwell DW (1971). The Description and Classification of Vegetation. Sidgwick \& Jackson, London.

44. Sher Z \& Khan ZU (2007). Floristic composition, life form and leaf spectra of the vegetation of Chagharzai Valley, District Buner. Pak J Pl Sci 13(1): 57 66.

45. Qureshi R, Shaheen H, Ilyas M, Wasim M \& Munir M (2014). Phytodiversity and plant life of Khanpur Dam, Khyber Pakhtunkhwa, Pakistan. Pak J Bot 46(3): 841- 849.

46. Qureshi R, Bhatti GR \& Shabbir G (2011). Floristic inventory of Pir Mehr Ali Shah
Arid Agriculture University research farm at Koont and its surrounding areas. Pak J Bot 43(3): 1679-1684.

47. Costa RC, Soares AF \& LimaVerde LW (2007). Flora and lifeform spectrum in an area of deciduous thorn woodland (caatinga) in northeastern, Brazil. Journal of Arid Environments 68: 237247.

48. Kar PK, Biswal AK \& Barik KL (2010). Floristic composition and biological spectrum of a grassland community of Rangamatia in the district of Mayurbhanj, Odisha. J Curr Sci 15(2): 465-469.

49. Guo QS, Wang XF, Bar G, Kang Y, Hong M, Pei SX \& Zhang FJ (2009). Life form spectra, leaf character, and hierarchical-synusia structure of vascular plants in Thuja sutchuehensis community. PubMed 20(9): 2057-2062.

50. Manhas RK, Singh L, Vasistha HB \& Negi M (2010). Floristic diversity of protected ecosystems of Kandi Region of Punjab, India. New York Science Journal 3 (4): 96-103.

51. Fazal H, Ahmad N, Rashid A \& Farooq S (2010). A Checklist of phanerogamic flora of Haripur Hazara, Khyber Pakhtunkhwa, Pakistan. Pak J Bot 42 (3): 15111522.

52. Hussain F, Ilyas M \& Takatsuki S (1997). Plant communities of Girbanr Hills, Swat district, northwestern Pakistan. Ecol Rev 23: 247-60.

53. Batalha MA \& Martins FR (2002). Lifeform spectra of Brazilian Cerrado sites. Flora, Morphology, Distribution, Functional Ecology of Plants 197(6): 452-460.

54. Saxina AK, Pandey TP \& Singh JS (1987). Altitudinal variation in the vegetation of Kaumaun Himalaya. Perspective in Env Bot 44-66.

55. Batalha MA \& Martins FR (2004). Floristic frequency, and vegetation life form spectra of a Cerrado site. Braz J Biol 64 (2): 203-209. 
56. Borchert R, Rivera G \& Hagnauer W (2002). Modification of vegetative phenology in a tropical semi-deciduous forest by abnormal drought and rain. Biotropica 34: 27-39.

57. Borchert R, Meyer SA, Felger RS \& Porter-Bolland L (2004). Environmental control of flowering periodicity in Costa Rican and Mexican tropical dry forests. Global Ecology and Biogeography 13: 409-425.

58. Zhang X, Tarpley D \& Sullivan JT (2007). Diverse responses of vegetation phenology to a warming climate. Geophys Res Lett 34: 1940-45.

59. Rocha PLBD, Queiroz LPD \& Pirani JR (2004). Plant species and habitat structure in a sand dune field in the Brazilian Caatinga: a homogeneous habitat harboring an endemic biota. Revista Brasil. Bot 27(4): 739-755.

60. Kikim A \& Yadava PS (2001). Phenology of tree species in subtropical forests of Manipur in north eastern India. Tropical Ecology 42: 269-276.

61. Malik ZH \& Malik NZ (2014). Phenological patterans among the vegetation of Ganga Chotti and Bedori hills in a moist temperate to alpine forests. Int J Bio Cons 6(6): 444-451.

62. Forrest JD, Inouye $\mathrm{W} \&$ Thomson $\mathrm{D}$ (2010). Flowering phenology in sub alpine meadows. Does climate variation influence community co-flowering patterns. Ecol 9(2): 431- 440.

63. Adrian MI, Tansey RC, Smithers RJ \& Phillimore AB (2015). Predicting a change in the order of spring phenology in temperate forests. Globle change biology 21(7): 2603-2611.

64. Nautiyal MC, Nautiyal BP \& Prakash V (2001). Phenology and growth form distribution in an alpine pasture at Tungnath, Garhwal Himalaya. Mountain Research and Development 21(2): 177183.

65. Dar MEUI \& Malik ZH (2009). A Floristic List and Phenology of Plant Species of Lawat Area District Neelum, Azad Jammu and Kashmir, Pakistan. Int J Bot 5(2): 194-199.

66. Golluscio RA, Oesterheld M \& Aguiar MR (2005). Relationship between phenology and life form: a test with 25 Patagonian species. Ecography 28: 273282

67. Huang J, Tardif JC, Bergeron Y, Denneler B, Berninger F \& Girardins MP (2010). Radial growth response of four dominant boreal tree species to climate along a latitudinal gradient in the eastern Canadian boreal forest. Global Change Biology 16: 711-731.

68. Nath AJ, Das G \& Das AK (2008). Vegetative phenology of three bamboo species in subtropical humid climate of Assam. Tropical Ecology 49(1): 85-89. 